Théologiques

Théologiques

\title{
Quand notre rapport au cosmos n'est plus tout à fait le même
}

\section{Robert David}

Volume 9, numéro 1, printemps 2001

Les cosmologies

URI : https://id.erudit.org/iderudit/005684ar

DOI : https://doi.org/10.7202/005684ar

Aller au sommaire du numéro

\section{Éditeur(s)}

Faculté de théologie de l'Université de Montréal

\section{ISSN}

1188-7109 (imprimé)

1492-1413 (numérique)

Découvrir la revue

Citer ce document

David, R. (2001). Quand notre rapport au cosmos n'est plus tout à fait le même. Théologiques, 9(1), 3-15. https://doi.org/10.7202/005684ar d'utilisation que vous pouvez consulter en ligne.

https://apropos.erudit.org/fr/usagers/politique-dutilisation/ 


\section{Quand notre rapport au cosmos n'est plus tout à fait le même}

Robert DAVID

Faculté de théologie

Université de Montréal

À quelques semaines du dépôt final des articles du présent dossier de THÉOLOGIQUES, trois événements apparentés à la problématique générale ont retenu notre attention. Chacun d'eux témoigne, à sa façon, d'une facette de la situation dans laquelle nous nous trouvons au seuil du troisième millénaire quant à notre rapport au cosmos et à ses représentations.

\section{Trois événements significatifs}

Jeudi 23 novembre 2000. Près de 350 personnes sont réunies dans l'un des plus grands amphithéâtres de la plus importante université francophone d'Amérique du nord. Au programme : un débat entre un tenant de la thèse créationniste et un professeur de sciences biologiques de l'Université de Montréal inscrit dans la foulée des thèses évolutionnistes. Comme il faut souvent s'y attendre dans ce type de débat, chacun expose ses perspectives en restant bien campé sur ses positions. Voudrions-nous demeurer le plus objectif possible et ouvert aux idées des autres, il faut admettre que certaines affirmations laissent parfois pantois. Nous ne retiendrons, à titre d'exemple, que celle-ci lancée avec le plus grand sérieux par le créationniste : la vie est apparue sur terre il y a 10,000 ans. La science, reconnaissons-le, ne réussit pas toujours à démontrer hors de tout doute le bien-fondé de toutes ses hypothèses. Mais force est d'admettre que les données géologiques et paléontologiques, pour ne mentionner que celles-là, nous ont au moins appris qu'il faut parler en terme de millions d'années. 
La thèse créationniste telle qu'exposée au cours de ce débat avait de quoi faire sourire, sans plus, n'eut été de l'accueil chaleureux réservé à celle-ci par près de la moitié des participant-e-s, la majorité ayant entre 20 et 35 ans, peut-être de futurs diplômé-e-s qui formeront la relève de demain. Tous ces partisans du créationnisme défendent une vision cosmologique qui, pour des motifs de conviction religieuse, va à l'encontre des plus élémentaires données scientifiques modernes ${ }^{1}$. Création spontanée, par un Dieu créateur omniscient et omnipotent, avec, comme toile de fond, une lecture littérale des textes bibliques, voilà pour l'essentiel le fondement de l'argumentation. Cet exemple illustre à lui seul la nécessité de réfléchir sur la question de nos représentations cosmologiques et, ce faisant, de leur impact sur nos discours anthropologique et théologique ${ }^{2}$.

Dans un registre situé à l'opposé des prémisses sous-tendant de tels débats, des essais de mise en forme d'une vision cosmologique ouverte au dialogue entre science et religion sont également tentés. C'est ainsi qu'à la fin mars 2001 un salon était organisé par le Centre d'étude des religions de l'Université de Montréal (CÉRUM). Y étaient regroupées une cinquantaine de personnes venues entendre une spécialiste du droit en bioéthique travaillant sur les problèmes éthiques liés aux recherches en génomique, un physicien ainsi qu'un mathématicien. Les trois ont présenté une réflexion à partir du thème suivant : Sciences et religions : contentieux et nouveaux dialogues. Le seul fait que des personnes provenant du monde des sciences dites exactes aient accepté de venir échanger leurs points de vue dans une faculté de théologie constituait déjà en soi un événement qui n'aurait pas été imaginable il y a quelques années. Cette ouverture au dialogue entre science et religion s'inscrit dans la mouvance d'un courant qui se développe de plus en plus dans la communauté scientifique, philoso-

1. Même le très conservateur état du Kansas est revenu, en février 2001, sur sa décision d'interdire la théorie de l'évolution dans son système d'enseignement public. (V. Dufour, «Kansas : la théorie de l'évolution a de nouveau droit de cité» dans Le Devoir, 27 février 2001, p. A5 ; voir aussi «Kansas Board Revives Teaching of Evolution " Los Angeles Times Feb. 15 2001, p. A10.).

2. La tenue de ce débat aura suscité de nombreuses réactions dans la communauté universitaire. Au moins cinq articles ont été publiés dans le journal FORUM entre le 4 décembre 2000 et le 21 janvier 2001. C'est la première fois, à notre connaissance, qu'autant d'articles traitaient du même sujet dans ce journal de l'Université de Montréal. 
phique et théologique. Il n'est pas étranger aux remises en question suscitées par les nouvelles cosmologies qui obligent à repenser nos présupposés épistémologiques et qui font éclater la sectorisation hermétique des divers champs du savoir et de la réflexion.

C'est dans cet esprit que le troisième événement s'insère. Il s'agit de la tenue d'un colloque organisé à l'UQÀM en avril 2001 par le programme d'éducation en religion et science du Canada (PERS) et par le Center for Theology and the Natural Sciences (CTNS) de Berkeley. La thématique (La science comme nouvelle religion : une question de métaphysique) abordée par des philosophe, métaphysicien, ingénieur biomédical et astrophysicien, a permis des échanges qui ont constitué une première dans la francophonie nord-américaine. Plusieurs questions y ont été soulevées, dont celles ayant trait aux discours religieux par rapport aux interrogations que posent les sciences pures, mais également celles concernant les limites des discours scientifiques quant aux notions de finalité et de sens. L'éclatement du paradigme de la mécanique newtonienne, maintes fois signalé par les conférenciers, fait place à une pluralité de modèles dont nous n'avons pas fini d'explorer les potentialités. Ici aussi, l'apport des nouvelles cosmologies n'est pas étranger à ce bouillonnement d'idées. Des questions sont renvoyées de part et d'autres qui obligent à penser nouvellement notre insertion au sein de ce nouvel univers ouvert et, ce faisant, à repenser également nos discours théologiques. Un astrophysicien posait un jour ce constat :

Le domaine de Dieu est celui du mystérieux et de l'invisible, celui de l'infiniment petit et de l'infiniment grand. Ce domaine n'appartient plus aujourd'hui exclusivement au théologien, il est aussi celui du scientifique. La science est là, qui accumule les découvertes et bouleverse les perspectives. Le théologien n'a plus le droit de rester indifférent. Confrontons les arguments religieux et philosophiques concernant l'existence de Dieu avec la nouvelle vision scientifique de l'univers. Examinons le face à face de Dieu et de la cosmologie moderne’3.

Les trois événements relatés sont une illustration partielle ${ }^{4}$ des situations qui prévalent au sein de nos sociétés contemporaines. On

3. Trinh Xuan Thuan, La mélodie secrète. Et l'homme créa l'univers, Paris, Gallimard, 1991, p. 297.

4. Parallèlement à ces exemples nous pourrions également faire prévaloir la somme considérable de livres et d'articles publiés depuis quelques décennies 
trouve encore des bastions de résistance qui voudraient retenir les schémas anciens. Pour certains la tentation est grande de s'y confiner. Pour d'autres, qu'on espérerait majoritaires, des défis nouveaux pointent à l'horizon et stimulent la réflexion et les échanges. C'est dans cette foulée que le présent numéro de THÉOLOGIQUES propose sa thématique autour des cosmologies.

\section{Quelques changements conceptuels majeurs}

Nous sommes entrés, depuis la première moitié $\mathrm{du} \mathrm{XX}^{\mathrm{e}}$ siècle, dans une période de transition marquée par des changements de représentations majeurs quant à notre rapport à la nature et au cosmos. Parmi ces changements, quatre nous semblent particulièrement significatifs puisqu'ils influencent de manière notable nos rapports au réel. Ils sont issus pour une bonne part des sciences physique et biologique et marquent souvent une rupture profonde avec les conceptions anciennes telles que prônées dans le domaine des sciences jusqu'à tout récemment ${ }^{5}$.

- A) HIER : La nature était considérée comme une structure simple, faite de substances réductibles à un nombre restreint de combinaisons possibles. La recherche scientifique s'engageait à identifier ces substances et à en isoler les quelques combinaisons.

- A') AUJOURD'HUI : La nature est préhendée ${ }^{6}$ comme système extrêmement complexe, composée d'une variété multiple d'interrelations dont les ramifications s'étendent des particules subatomiques aux entités occupant la biosphère et s'intégrant au mégacosme de l'univers intergalactique et, peut-être, extragalactique. Ici, le terme

touchant l'importance du dialogue entre science et religion et sur la nécessité d'en tenir compte dans l'élaboration d'une réflexion théologique contemporaine. Pour un palmarès régulier on consultera la revue Science and Spirit.

5. Avouons d'entrée de jeu que certaines recherches scientifiques pataugent encore dans la mouvance des anciens paradigmes, surtout celui du déterminisme mécanique. Il n'est qu'à écouter certains discours de chercheurs travaillant sur le génome ou sur le clonage pour s'en rendre compte.

6. Le terme " préhendé " renvoie au mot "préhension » dans le système de la pensée de A.N. WhiteHEAD tel que développé dans Procès et réalité. Essai de cosmologie, Paris, Gallimard (Bibliothèque de philosophie), 1995 (original anglais 1929), principalement dans la troisième partie " La théorie des préhensions » p. 353-419. 
essentiel est interrelations. Plus question d'analyser un aspect isolé du réel comme fin en soi ; chaque élément fait partie intégrante d'un réseau infini, affecte et est affecté par l'ensemble.

- B) HIER : le monde était vu comme déterminé mécaniquement, prévisible selon certaines lois pouvant s'appliquer à tous les domaines. C'est le modèle de la mécanique newtonienne, qui, reconnaissons-le, prévaut encore dans plusieurs domaines de recherche.

- B') AUJOURD'HUI : le monde est constitué par un croisement entre la chance et certaines uniformités statistiques sans que l'on puisse parler de déterminisme au niveau du microcosme (c'est du moins ce que suggèrent les théories quantiques) et imprévisible au niveau du macrocosme (biologique) vue la complexité des chaînes causales. Le démon de Laplace s'y trouve relégué aux oubliettes.

- C) HIER : le monde naturel était plutôt considéré comme statique, fermé et complet, sans place pour la nouveauté et l'imprévisible. C'était le lieu idéal pour proclamer le fameux proverbe de Qohéleth : «Il n'y a rien de nouveau sous le soleil».

- C') AUJOURD'HUI : le monde naturel est dynamique, en continuel processus de nouveauté, incomplet. Il possède un potentiel de changement incalculable dont l'ouverture sur le futur constitue l'une des plus importantes caractéristiques. Cette ouverture fait place à la nouveauté, à la créativité, à l'inattendu irréductible aux seules données causales ${ }^{8}$.

7. La cosmologie du procès (process) exprime cette réalité par le principe de relativité qui se luit comme suit dans Procès et réalité, p. $74:$ :La potentialité d'être un élément dans une concrescence réelle d'une pluralité d'entités en une actualisation unique est le seul caractère métaphysique général attaché à toutes les entités actuelles et non actuelles; et chaque élément de son univers est compris dans chaque concrescence. En d'autres termes, il appartient à la nature d'un « être » d'être un potentiel pour chaque « devenir ».

8. Toujours chez Whitehead, cette idée recoupe l'une des catégories ultime de son système, celle de créativité. Il en dit ceci : "La créativité » est l'universel des universaux qui caractérise le fait ultime. C'est ce principe ultime par lequel la pluralité, qui est l'univers pris en disjonction, devient l'occasion actuelle unique, qui est l'univers pris en conjonction. ... La « créativité » est le principe de la nouveauté » (ibid., p. 72). 
- D) HIER : le monde semblait décomposable en sous-unités dont on pouvait atteindre les fondations, ce qui s'est traduit par une forme d'atomisme dans les approches scientifiques.

- D') AUJOURD'HUI : le monde laisse présager un sens du mystère dans ce qui est connu alors que l'inconnu atteint des dimensions impossibles à imaginer. Les découvertes qui se multiplient donnent l'impression que les fondations de la connaissance ne seront jamais atteintes et pousseront toujours vers l'avant les chercheurs qui s'engageront dans leurs recherches.

D'autres changements pourraient être évoqués ${ }^{9}$, mais les quatre retenus sont suffisamment importants pour que l'on sente dès à présent que les règles du jeu sont modifiées en profondeur. Il nous faut les prendre au sérieux sans quoi nous risquons d'être mis hors-jeu. Ce constat doit aussi s'appliquer aux domaines religieux et théologique. Si les représentations scientifiques d'hier ont pu permettre de concevoir l'entité divine comme un grand horloger ayant mis en place les rouages d'une mécanique qui se suffisait à elle-même (permettant du même coup de nous passer éventuellement de l'horloger), les nouveaux paradigmes s'apparentent davantage à l'idée de maître de jeu ${ }^{10}$ qui, tout en respectant certaines règles élémentaires, se laisse prendre au jeu de l'inattendu, de l'imprévisible et d'une certaine improvisation.

\section{Vers un réenchantement}

L'une des conséquences d'une vision cosmologique apparentée à la mécanique newtonienne fut le "désenchantement du monde " (Weber) qu'elle provoqua. Si l'on pouvait remonter aux fondements des lois gouvernant l'ensemble du cosmos on pouvait du même coup rendre compte de l'ensemble du fonctionnement du monde et du cosmos. Connaître un rouage équivalait à connaître la machine. On a fait miroiter, pendant plus d'une génération, l'illusion que la science pouvait tout expliquer, qu'il suffisait d'un peu de patience pour que l'on finisse par

9. L'ouvrage de Charlene SPRETNAK, The Resurgence of the Real. Body, Nature and Place in a Hypermodern World, N.Y., Routledge, 1999, s'intéresse à ces situations nouvelles et trace la voie à une réflexion originale et pertinente.

10. On lira à ce propos l'intéressant ouvrage de François Euvé, Penser la création comme jeu, Paris, Cerf (Cogitatio Fidei 219), 2000. 
venir à bout de toutes les entraves à la connaissance parfaite. Cette promesse a déçu car elle n'a pu livrer les fruits escomptés. Non pas à cause des limites de nos connaissances et de nos moyens d'investigation, mais parce que le réel tel qu'il se déploie est incommensurablement plus complexe que ce à quoi on pensait pouvoir le réduire. Devant cette complexité sans cesse grandissante, des chercheurs en arrivent à poser un regard réenchanté sur le monde ${ }^{11}$ et, pour certains, à laisser la porte ouverte à une forme de questionnement religieux. Quelques-uns privilégieront les rapprochements avec les intuitions formulées dans les religions orientales ${ }^{12}$ tandis que d'autres ouvriront le dialogue au sein de la tradition chrétienne ${ }^{13}$. Quelle que soit la porte d'entrée, ces incursions de scientifiques dans le domaine des questions de sens marquent un tournant important et significatif. Pendant des siècles on a voulu sépa-

11. Dans son dernier ouvrage paru en français, Les mystères de l'arc-en-ciel. Paris, Bayard, 2000, p. 8, le biologiste anglais Richard Dawkins invite ses lecteurs à se laisser réenchanter, émerveiller, à retrouver la poésie qu'inspirent les diverses découvertes scientifiques : "l'ouvrage entend d'abord promouvoir la bonne science poétique : je n'entends pas par là, bien sûr, une science formulée en vers, mais inspirée par un sens poétique de l'émerveillement ». Il reste cependant fidèle à lui-même en écartant l'univers religieux de son discours.

12. On sent cette tendance, entre autres, dans les ouvrages de deux astrophysiciens : Hubert REEVES, L'heure de s'enivrer, Paris, Seuil, 1986, et dans celui de Trinh Xuan THuAN, La mélodie secrète. Et l'homme créa l'univers, Paris, Gallimard, 1991. Il revient sans doute à Fritjof Capra d'avoir le plus clairement exprimé ses positions à ce chapitre dans son livre Le Tao de la physique, Paris, Sand, 1985.

13. Les ouvrages consacrés aux rapprochements entre les nouvelles données cosmologiques et la théologie chrétienne sont nombreux. Nous n'en citons ici que quelques-uns qui ont particulièrement marqué notre propre réflexion : Jacques ARNOULD, La théologie après Darwin, Paris, Cerf, 1998 ; Ian BARbour, Religion in an Age of Science, San Francisco, Harper, 1990 ; Edmond Blattchen et all., Où va Dieu ?, Bruxelles, Éditions Complexe, 1999 ; François Euvé, Penser la création comme jeu, Paris, Cerf (Cogitatio Fidei 219), 2000 ; Adolphe GESCHÉ, Dieu pour penser. Tome IV. Le Cosmos, Paris, Cerf, 1994 ; Pierre Gisel et Lucie KAENNEL, La création du monde. Discours religieux, discours scientifique, discours de foi, Genève, Labor et Fides, 1999 ; David R. GRIFFIN, ed., The Reenchantment of Science. Postmodern Proposals, Albany, SUNY Press, 1988 ; Dominique LAMBERT, Sciences et théologie. Les figures d'un dialogue, Bruxelles, Lessius, 1999 ; 
rer les discours scientifique, philosophique et théologique en les cloisonnant dans leur propres univers conceptuel. Les nouveaux paradigmes ouverts par l'entrée en scène de nouvelles représentations cosmologiques laissent présager des mises en commun qui, sans nier les différences de niveaux de langage et de préoccupations, n'en acceptent pas moins les défis de l'échange, du dialogue et de l'essai de compréhension mutuelle.

\section{Quand l'ancien ne fonctionne plus}

L'impact des nouvelles représentations cosmologiques se fait sentir dans plusieurs champs du savoir. La réflexion théologique ne peut échapper à celui-ci. La théologie se trouve nécessairement confrontée, elle aussi, à ces nouvelles données. Elle ne peut plus se contenter de répéter les vieux schèmes et s'en tenir à l'héritage biblique et à celui de la Tradition. Bien sûr doit-on continuer de puiser dans les intuitions léguées par ceux et celles qui nous ont précédés, mais l'heure est aussi à la reformulation, à la mise à niveau et, d'une certaine manière, à l'émergence de représentations, d'images et de concepts totalement nouveaux qui se démarquent singulièrement de l'héritage. Que nous le voulions ou non, la théologie a construit son imaginaire cosmologique en bonne partie sur la base des affirmations contenues dans les textes bibliques. À ce chapitre, l'Ancien Testament et le Nouveau partagent les mêmes images et les mêmes conceptions. Or, ces images, en plus d'appartenir à d'autres cultures que les nôtres, relèvent également d'autres époques.

La lecture du Psaume 104 révèle à elle seule l'écart qui nous sépare de ces cosmologies. On y décèle un condensé de quelques-unes des affirmations centrales de la cosmologie ancienne, affirmations qui sous-tendent l'édifice conceptuel théologique des auteurs bibliques, de la plupart de

Jürgen Moltmann, Dieu dans la création. Traité écologique de la création, Paris, Cerf (Cogitatio Fidei 146), 1988 ; Arthur PEACOCKE, Theology for a Scientific Age. Being and Becoming - Natural, Divine and Human, Minneapolis, Fortress Press, 1993 ; Ted PeTERs, Cosmos as Creation. Theology and Science in Consonance, Nashville, Abingdon Press, 1989 ; Gérard SIEGWalt, Dogmatique pour la catholicité évangélique. Système mystagogique de la foi chrétienne. III/1 Cosmologie Théologique : Sciences et philosophie de la nature. III/2 Cosmologie théologique: Théologie de la création, Paris/Genève, Cerf, Labor et Fides, 1996 et 2000. 
ceux qui ont élaboré la théologie classique, et qui continuent d'être présentes dans plusieurs discours théologiques aujourd'hui. On retrouve dans ce psaume des images familières au lecteur de Gn 1, qu'il s'agisse de la voûte céleste tendue comme une toile (v. 2) ou des eaux qui couvraient la terre (vv. 6-7), centre de cet univers. Nous ne pouvons plus recevoir ce psaume, et toute la cosmologie qui s'y exprime, sans ressentir un certain malaise, pour ne pas dire un malaise certain.

Nous savons maintenant que la terre n'est pas "inébranlable à jamais et toujours » (v.5). Elle a un commencement, une histoire et une fin. Elle est menacée à intervalles réguliers par des cataclysmes qui peuvent changer en profondeur sa configuration et mettre en péril la survie de l'ensemble de ce qui y vit. On peut penser bien entendu aux menaces associées aux activités humaines (pollution, conflit ou accident nucléaire, déforestation, etc.), mais il en existe aussi qui sont dues aux conditions cosmiques dans lesquelles gravite notre planète. L'oscillation galactique devrait nous mettre en situation d'impact météorique majeur dans un avenir plus ou moins rapproché, impact qui aura des effets aussi dévastateurs que celui qui a mis fin au règne des dinosaures il y a quelques millions d'années. L'identification de supervolcans a alerté les scientifiques quant à la menace d'une destruction massive de toute vie sur terre ${ }^{14}$. Nous savons, d'ores et déjà, que dans quelques milliards d'années, alors que le soleil se transformera en supergéante rouge, il grillera la terre comme un fétu de pailles. Nous ne vivons pas sur une planète inébranlable. Les humains peuvent, et vont, disparaître. Comment penser théologiquement dans ce contexte?

Il faut également tenir compte de nos nouveaux rapports au temps et à l'espace qui nous font nous situer dans un univers totalement éclaté, sans commune mesure avec ces réalités telles que décrites dans les textes bibliques et telles que conçues jusqu'au $\mathrm{XVIII}^{\mathrm{e}}$ siècle $^{15}$. Le

14. La dernière éruption d'un supervolcan, le Toba dans l'île de Sumatra, remonte à 74,000 ans et avait une puissance équivalent à 10,000 fois celle du Mont Ste-Helen aux États-Unis. Les scientifiques estiment que le parc de Yellowstone cache en son sein un supervolcan dont ils évaluent la fréquence des éruptions à 600,000 ans. Sa dernière remonterait à 640,000 ans ...

15. Voir à ce sujet les réflexions proposées dans Robert DAVID, "L'indéterminisme biblique. Une proposition du procès » dans Théoforum, 32 (2001), p. 63-82 (66-80). 
monde sublunaire a été anéanti avec les découvertes de Tycho Braché ${ }^{16}$ et les dimensions de l'univers connu atteignent des proportions impossibles à se représenter ${ }^{17}$ (entre 12 et 13.5 milliards d'années lumière). Les travaux de Prigogine et de l'école de Bruxelles nous situent également dans de nouveaux paramètres concernant l'indéterminisme et l'irréversibilité du temps ${ }^{18}$ allant jusqu'à suggérer qu'il n'y a peut-être pas de commencement du temps ${ }^{19}$ (à distinguer alors du commencement de notre univers).

Comment situer Dieu dans ces nouveaux contextes et comment le dire dans ces nouvelles catégories impensables jusqu'au début du $\mathrm{XX}^{\mathrm{e}}$ siècle ? Le recours à la révélation spéciale et à la Tradition, pour important qu'il soit, ne peut plus être le seul invoqué. Il faut repenser les images, les représentations, le vocabulaire, les idées. Il ne s'agit pas de changements mineurs où l'on se contenterait d'un maquillage superficiel pour se refaire une beauté. C'est à une chirurgie majeure que nous sommes conviés. Par rapport à tout ce qui nous a précédés, les nouvelles données cosmologiques nous situent ailleurs, autrement. L'ensemble des savoirs humains se trouve interpellé, y compris la théologie.

\section{Les contributions de ce numéro}

Les cinq articles consacrés à la thématique du présent numéro s'inscrivent dans cette recherche de nouveauté dans la continuité. Pierre Gisel

16. Jan Marejko signale bien cette révolution majeure dans son article « Le roi du technocosme est nu ", dans Maurice WeYEMBERGH - Gilbert HOTTOIS, dir., Temps cosmique, histoire humaine, Paris, J. Vrin, 1996, p. 83-115. 17. Et c'est sans parler de la théorie des univers plats telle que proposée aujourd'hui dans la communauté scientifique. Voir à ce propos : 1) http:// www.lbl.gov/Science-Articles/Archive/boomerang-flat.html ; 2) http :// www.lbl.gov/Science-Articles/Archive/boomerang-flat.html. Certains avancent aussi l'idée d'univers parallèles...

18. Deux ouvrages généraux de Ilya Prigogine exposent ses recherches et leurs conséquences sur l'irréversibilité du temps : avec Isabelle STENGERS, $\mathrm{La}$ nouvelle Alliance. Métamorphose de la science, Paris, Gallimard (Folio/ Essais), 1986 ; La fin des certitudes. Temps, chaos et les lois de la nature, Paris, Odille Jacob (Opus), 1998.

19. Ilya PRIGogine, "La quête de la certitude» dans Maurice WeYeMBERGH - Gilbert HOTTOIS, dir., Temps cosmique, histoire humaine, Paris, J. Vrin, 1996, p. 14. 
ouvre la discussion en rappelant d'abord que le christianisme n'en est pas à ses premiers changements de paradigmes. Il a eu à se repenser au fur et à mesure qu'émergeaient ces changements. Après quelques notices scientifiques, Gisel énonce brièvement quelques points majeurs qui ressortent de la tradition biblique d'abord, puis de la tradition chrétienne. Depuis Augustin en passant par Thomas d'Aquin et Vatican I, le christianisme s'est souvent trouvé confronté à des représentations du monde qu'il ne partageait pas nécessairement. Ces conflits ne sont pas étrangers à la rupture qui s'est imposée entre science et foi, mais le temps est venu de rétablir les ponts. Encore faut-il s'entendre sur le type de complémentarité qui peut exister entre le scientifique et le religieux. Gisel en explore quelques-uns avant de poser la question du statut de la théologie dans ces différentes composantes.

Jean Proulx, philosophe et poète, se donne comme objectif de ranimer la dimension cosmologique de la théologie chrétienne. Il rejoint en cela une observation de Gisel. Les deux en effet reconnaissent que la théologie a eu tendance à privilégier la thématique du salut (centrée sur l'humain) au détriment de celle de la création (ouverte sur le cosmos). Lui aussi insiste sur le fait que les nouvelles cosmologies et la physique nous obligent à repenser notre place et notre rôle au sein du tout insécable et organique. La cosmologie scientifique offre de nouveaux horizons mais ne les épuise pas tous. Ses champs d'investigation restent limités à l'observable, au vérifiable. Sont ouverts, le domaine de la cosmologie ontologique et de la théologie dans sa dimension cosmologique. En acceptant d'accueillir d'autres dimensions que celle de la science, Proulx tente des rapprochements entre ce que l'on pourrait qualifier de trinité théologique et de «trinité» de la nouvelle physique (énergie, intelligibilité, liaison) et propose une lecture différente de lieux théologiques traditionnels (Dieu, Christ, Église, liturgie). Pour Proulx, Dieu inhabite l'univers. L'esprit cosmique est l'âme du monde tout comme le monde peut être dit «corps de Dieu». Par ces catégories Proulx trace certaines balises qu'il vaudra la peine d'explorer plus à fond à l'avenir.

Dans un registre un peu différent, mais qui emprunte les mêmes constats quant à la nécessité de rapprochements entre science et religion, Paul Allen s'intéresse à une thématique particulière, celle de l'auto-transcendance. C'est parce que de nouvelles sciences (neurosciences, biologie évolutive, cosmologie et astrophysique) posent de nouvelles questions quant à l'identité humaine, que la philosophie et la 
théologie se doivent d'interroger de façon neuve cette notion d'autotranscendance. Pour Allen, cette notion en est une éminemment théologique qui émerge de la cosmologie elle-même. Ses réflexions le conduisent sur la voie d'intuitions formulées autour d'un thème très présent dans les discussions entre science et religion, celui du principe anthropique. Il utilise ce principe à titre d'exemple pour montrer comment une théorie cosmologique se dirige elle-même, presque de façon autonome, dans le domaine philosophique et peut trouver sa place dans celui de la théologie. La cosmologie a ainsi peut-être ouvert un chemin de réflexion, mais elle doit aussi laisser place au surplus de sens qui existe au-delà des limites de la recherche scientifique.

Christian Downs offre de son côté une contribution particulière à l'un des thèmes abordés par Jean Proulx auparavant, celui de l'acosmisme théologique. Euvrant actuellement à une recherche doctorale dans laquelle il travaille principalement sur les ouvrages de G. Siegwalt (voir note 13), Downs s'inscrit en faux contre le projet de démythologisation tel que mit de l'avant par l'Institut de recherches herméneutiques et systématiques de Neuchâtel. Au compartimentage et au dualisme inhérents aux travaux de l'Institut, il préfère l'holisme dialectique du monde vécu dans sa diversité, tel que le propose Siegwalt. Comme les autres contributions du présent numéro, il reconnaît la partialité des sciences empirico-formelles, ce qui invite à laisser la porte ouverte à l'invisible, l'inobservable, à l'aspect ontologique de l'être comme fondement et fin. Le mythe a sa place dans un monde marqué par l'empiricoformel, à condition de ne pas réduire le réel à l'empirico-formel.

C'est dans le cadre de cette ouverture au mythe et à sa possibilité d'existence qu'il faut accueillir la contribution de John Grimm. Celuici nous fait sortir des sentiers «scientifiques» empruntés jusqu'à maintenant pour nous inviter à pénétrer au cœur des cosmologies et des traditions mystiques des peuples aborigènes d'Amérique du Nord. On retrouve dans ces traditions mystiques des intuitions que les sciences modernes ont remises au calendrier des réflexions contemporaines. Que l'on pense en particulier aux champs conceptuels relationnels entre les parties et le tout, entre la communauté, les personnes et les écosystèmes. Les lieux d'ancrages des expériences mystiques amérindiennes et des sciences empirico-formelles, bien que différents, convergent cependant vers l'idée de participation active et intégrante avec l'ensemble du cosmos. 
Les articles du présent numéro sont une invitation à chacun et chacune de tenter le travail d'intégration, de réflexion et de mise à neuf qu'impose le dialogue ouvert entre cosmologies modernes, philosophie et théologie. Comme le monde avance et change, il n'est plus possible de camper sur des positions théologiques figées et stagnantes. Le dynamisme qui gouverne le cosmos et le pousse en avant doit également s'insinuer dans nos représentations théologiques. 